\title{
HEREDITARY ORDERS
}

\author{
BY \\ MANABU HARADA(1)
}

Introduction. Let $R$ be an integral noetherian domain with field of quotients $K$. Let $\Sigma$ be a semi-simple $K$-algebra with finite dimension over $K$. By an order over $R$ we mean a subring $\Lambda$ in $\Sigma$ such that $\Lambda$ is a finitely generated $R$-module which spans $\Sigma$ over $K$, and that $\Lambda$ contains the identity element in $\Sigma$.

A ring $\Lambda$ will be called hereditary if every left and right ideal in $\Lambda$ is $\Lambda$-projective.

In the paper of Auslander and Goldman [3], they have obtained the following fact: Let $R$ be a discrete, rank one valuation ring, and $\Sigma$ a central simple $K$ algebra; then $\Lambda$ is maximal if and only if $\Lambda$ is hereditary and the radical of $\Lambda$ is a unique maximal ideal in $\Lambda$ (see Corollary 3.5). Furthermore, they have given a nonmaximal hereditary order in which there are two maximal two-sided ideals and over which there are two maximal orders (cf. [9]).

This fact suggests that there are some relations between maximal orders containing $\Lambda$ and maximal two-sided ideals.

The purpose of this paper is to investigate such a relationship and to give analogous properties of hereditary orders over a Dedekind domain to classical properties of maximal orders.

In $\S 1$, we give a fundamental theorem: There is a one-to-one correspondence between orders containing an hereditary order $\Lambda$ and idempotent ideals in $\Lambda$. Using this fact, we shall reduce, in $\S 2$, problems to the case where $R$ is a Dedekind domain and $\Sigma$ is a central simple $K$-algebra.

In $\S \S 3,4,5$, and 6 , we study hereditary orders over a discrete, rank one valuation ring. We shall give a complete description of orders containing an hereditary order $\Lambda$, and some relations of orders containing $\Lambda$. Furthermore, we see that the associated division rings of simple components of $\Lambda / N$ do depend only on $\Sigma$, not on $\Lambda$, where $N$ is the radical of $\Lambda$. We shall consider in $\S 5$ some criteria of hereditary order.

In $\S 6$, we consider a group structure of two-sided ideals with respect to $\Lambda$ and in $\$ 7$, generalizing the above results to the case of a Dedekind ring, we obtain

Presented to the Society, August 29, 1962; received by the editors March 28, 1962.

(1) The author wishes to express his gratitude to Professor Auslander and Professor Rim for their guidance and encouragement prior to the preparation of this paper. This paper is based on the author's doctoral dissertation at Brandeis University, Waltham, Massachusetts. This work was partially supported by the National Science Foundation. 
that the set of inversible two-sided ideals with respect to $\Lambda$ is an abelian group which is a direct product of cyclic group.

Some of our results are already given in $[1 ; 2 ; 3 ; 10]$.

1. Fundamental theorem in an hereditary order. Throughout this section, $R$ stands for a commutative noetherian domain, and $K$ its quotient field, unless stated to the contrary.

Let $\Sigma$ be a semi-simple $K$-algebra with finite dimension over $K$. By an order over $R$ we mean a subring $\Lambda$ such that $\Lambda$ is a finitely generated $R$-module containing the identity element in $\Sigma$, which spans $\Sigma$ over $K$. Hence, $\Lambda$ contains a $K$-basis of $\Sigma$ and $\Lambda$ is a left and right noetherian ring.

Let $\Lambda_{1}$ and $\Lambda_{2}$ be orders in $\Sigma$. Then subset $C_{\Lambda_{2}}\left(\Lambda_{1}\right)$ in $\Sigma$ consisting of all elements $x$ in $\Sigma$ such that $\Lambda_{1} x \subseteq \Lambda_{2}$ is a left $\Lambda_{1}$ - and right $\Lambda_{2}$-module. We call $C_{\Lambda_{2}}\left(\Lambda_{1}\right)$ the (right) conductor of $\Lambda_{1}$ with respect to $\Lambda_{2}$. Similarly, we can define the left conductor and we shall denote it by $D_{\Lambda_{2}}\left(\Lambda_{1}\right)$. If we fix $\Lambda_{2}$, then we denote briefly $C_{\Lambda_{2}}\left(\Lambda_{1}\right)$ by $C\left(\Lambda_{1}\right)$. We shall use frequently the following well-known result (Lemma 1.1) in this paper and so we recall the definition of trace ideal.

Let $S$ be any ring and $E$ a finitely generated left $S$-module. By the trace mapping $\tau$ of $E$ we mean the two-sided $S$-homomorphism of $E \otimes_{T} \operatorname{Hom}_{S}(E, S)\left({ }^{2}\right)$ to $S$ by setting $\tau(e \otimes f)=f(e)$, where $T=\operatorname{Hom}_{S}(E, E), e \in E$ and $f \in \operatorname{Hom}_{S}(E, S)$. Then the image $\tau_{S}(E)$ is the two-sided ideal generated by the image of $f$ where $f$ runs through all elements in $\operatorname{Hom}_{S}(E, S)$. Therefore, we obtain that $\operatorname{Hom}_{S}(E, S)$ $=\operatorname{Hom}_{S}\left(E, \tau_{S}(E)\right)$. We call $\tau_{S}(E)$ the trace ideal of $E$.

The following lemma is given in [3, Appendix]:

LEMMA 1.1. Let $S$ be a ring and $E$ a finitely generated left $S$-module. Let $\mathrm{T}=\operatorname{Hom}_{S}(E, E)$. Then (1) If $\tau_{S}(E)=S, E$ is a finitely generated projective T-module and $S=\operatorname{Hom}_{T}(E, E)$. (2) If $E$ is a finitely generated projective $S$ module, then $\tau_{S}(E) E=E$ and $\tau_{T}(E)=T$.

LEMMA 1.2. Let $\Lambda_{1} \supseteq \Lambda_{2}$ be orders in $\Sigma$ and let $E_{1}$ and $E_{2}$ be left $\Lambda_{1}$-modules such that $E_{2}$ is $R$-torsion free. Then we have $\operatorname{Hom}_{\Lambda_{1}}\left(E_{1}, E_{2}\right)=\operatorname{Hom}_{\Lambda_{2}}\left(E_{1}, E_{2}\right)$.

Proof. It is clear that $\operatorname{Hom}_{\Lambda_{1}}\left(E_{1}, E_{2}\right) \subseteq \operatorname{Hom}_{\Lambda_{2}}\left(E_{1}, E_{2}\right)$. By the definition of an order, we can find an element $r \neq 0$ in $R$ such that $r \Lambda_{1} \subseteq \Lambda_{2}$. For $f \in \operatorname{Hom}_{\Lambda_{2}}\left(E_{1}, E_{2}\right)$, $e_{1} \in E_{1}$ and $\lambda_{1} \in \Lambda_{1}$, we have that $f\left(r \lambda_{1} e_{1}\right)=r f\left(\lambda_{1} e_{1}\right)$, and $f\left(r \lambda_{1} e_{1}\right)=r \lambda_{1} f\left(e_{1}\right)$. Since $E_{2}$ is $R$-torsion free, $f\left(\lambda_{1} e_{1}\right)=\lambda_{1} f\left(e_{1}\right)$.

LEMMA 1.3. Let $\Lambda \subset \Gamma$ be orders and $E$ a finitely generated left $\Gamma$-module and $R$-torsion free. If $E$ is $\Lambda$-projective, then $E$ is $\Gamma$-projective.

(2) For a left (right) $S$-module $E$, every element of a ring of endomorphism of $E$ as a left (right) $S$-module operates on $E$ from the right (left) side. 
Proof. We have the following commutative diagram:

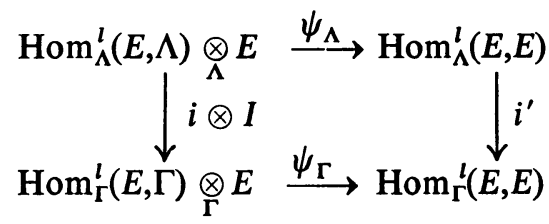

where $i^{\prime}$ is the identity mapping by Lemma 1.2 and $i: \operatorname{Hom}_{\Lambda}^{l}(E, \Lambda) \rightarrow \operatorname{Hom}_{\Lambda}^{l}(E, \Lambda)$ $=\operatorname{Hom}_{\Gamma}^{l}(E, \Gamma)$, and $\psi_{\Lambda}(f \otimes e)\left(e^{\prime}\right)=f\left(e^{\prime}\right) e, f \in \operatorname{Hom}_{\Lambda}^{l}(E, \Lambda)$ and $e, e^{\prime} \in E$. By assumption and [5, p. 123, Proposition 3.1], $\psi_{\Lambda}$ is epimorphic, and hence $\psi_{\Gamma}$ is epimorphic, which implies $E$ is $\Gamma$-projective.

CoROllary 1.4. Let $\Lambda$ be an hereditary order in $\Sigma$. Then every order containing $\Lambda$ is also hereditary.

Proof. Since there exists an element $r \neq 0$ in $R$ such that $\Gamma r \subseteq \Lambda$, every left (right) ideal in $\Gamma$ is $\Lambda$-isomorphic to a left (right) ideal in $\Lambda$. Hence, $\Gamma$ is hereditary.

Lemma 1.5. Let $S$ be any ring and $A$ a two-sided ideal in $S$ such that $A$ is left $\Lambda$-projective. Then $\left({ }^{3}\right) \tau_{s}^{l}(A)=A$ if and only if $A$ is idempotent, i.e., $A^{2}=A$.

Proof. If $\tau_{S}^{l}(A)=A$, then by assumption, we have $A=\tau_{S}^{l}(A) A=A^{2}$ by Lemma 1.1. Conversely, if $A=A^{2}$, then for any element $f$ in $\operatorname{Hom}_{S}^{l}(A, S)$, we have $f(A)=f(A A)=A f(A) \subseteq A$, which means $\tau_{S}^{l}(A) \subseteq A$. It is clear for any ideal $A$ that $\tau_{S}^{l}(A) \supseteq A$.

From now on, when we fix an order $\Lambda$, then an ideal $A$ means a fractional two-sided ideal with respect to $\Lambda$, namely $A$ is a two-sided $\Lambda$-module in $\Sigma$ such that $A K=\Sigma$ and $A r \subseteq \Lambda$ for some $r \neq 0$ in $R$.

Let $A$ be an ideal in an order $\Lambda$ in $\Sigma$. Then $\operatorname{Hom}_{\Lambda}^{r}(A, A)=\{x \mid \in \Sigma, x A \subseteq A\}$ and we shall denote it by $\operatorname{End}_{\Lambda}^{r}(A)$. Since $A$ is a faithful $\Lambda$-module, $\operatorname{End}_{\Lambda}^{r}(A)$ is an order containing $\Lambda$.

Proposition 1.6. Let $\Lambda$ be an order over $R$ in the semi-simple K-algebra $\Sigma$, and $A$ an ideal in $\Lambda$. Then, (1) $C\left(\operatorname{End}_{\Lambda}^{r}(A)\right) \supseteq \tau_{\Lambda}^{l}(A)$. If $A$ is right $\Lambda$-projective, then $C\left(\operatorname{End}_{\Lambda}^{r}(A)\right)=\tau_{\Lambda}^{l}(A) \cdot(2) C(\Gamma)=\tau_{\Lambda}^{l}(C(\Gamma))$ for any order $\Gamma$ containing $\Lambda$. If $\Gamma$ is left $\Lambda$-projective, then $C(\Gamma)$ is right $\Lambda$-projective. Furthermore, if $C(\Gamma)$ is idempotent, then $\operatorname{End}^{r}(C(\Gamma))=\Gamma$.

Proof. (1) We can easily see by the same method as in the proof of Lemma 1.2 that $C\left(\operatorname{End}_{\Lambda}^{r}(A)\right) \supset \tau_{\Lambda}^{l}(A)$. If $A$ is right $\Lambda$-projective, then we have an isomorphism $\psi: A \otimes_{\Lambda} \operatorname{Hom}_{\Lambda}^{l}(A, \Lambda) \rightarrow \operatorname{Hom}_{\Lambda}^{l}\left(\operatorname{Hom}_{\Lambda}^{r}(A, A), \Lambda\right)$ by setting $\psi(a \otimes f) g=f(g(a))$, where $f \in \operatorname{Hom}_{\Lambda}^{l}(A, \Lambda), g \in \operatorname{Hom}_{\Lambda}^{r}(A, A)$, and $a \in A$. However, the right side is

(3) Let $F$ be functor of a category of left (right) $\Lambda$-modules to a category. We denote $F($ ) by $F^{l}()(F())$ if there is ambiguity. 
equal to $C\left(\operatorname{End}_{\Lambda}^{r}(A)\right)$, and for $\sigma \in C\left(\operatorname{End}_{\Lambda}^{r}(A)\right), \psi^{-1}(\sigma)=\Sigma a_{i} \otimes f_{i}$ and $\sigma=I \sigma$ $=\psi\left(\Sigma a_{i} \otimes f_{i}\right)(I)=\Sigma f_{i}\left(a_{i}\right) \in \tau_{\Lambda}^{l}(A)$, where $I$ is the identity in $\operatorname{End}_{\Lambda}^{r}(A) \subseteq \Sigma$.

(2) Since $C(\Gamma)$ is a left $\Gamma$-module, we have $\operatorname{End}_{\Lambda}^{r}(C(\Gamma)) \supseteq \Gamma$, and hence, $C(\Gamma) \subseteq \tau_{\Lambda}^{l}\left(C(\Gamma) \subseteq C\left(\operatorname{End}_{\Lambda}^{r}(C(\Gamma))\right) \subseteq C(\Gamma)\right.$. It is clear that $C(\Gamma)$ is isomorphic to $\operatorname{Hom}_{\Lambda}^{l}(\Gamma, \Lambda)$ as a two-sided $\Lambda$-module. Hence, $C(\Gamma)$ is a right projective $\Lambda$-module if $\Gamma$ is left $\Lambda$-projective. Furthermore, we assume that $C(\Gamma)$ is idempotent. Then $\operatorname{Hom}_{\Lambda}^{l}(C(\Gamma), C(\Gamma))=\operatorname{Hom}_{\Lambda}^{l}(C(\Gamma), \Lambda)=\operatorname{Hom}_{\Lambda}^{r}\left(\operatorname{Hom}_{\Lambda}^{l}(\Gamma, \Lambda), \Lambda\right)$. Since $\Gamma$ is left $\Lambda$ projective, $\operatorname{Hom}_{\Lambda}^{r}\left(\operatorname{Hom}_{\Lambda}^{l}(\Gamma, \Lambda), \Lambda\right) \approx \operatorname{Hom}_{\Lambda}^{r}(\Lambda, \Lambda) \otimes_{\Lambda} \Gamma \approx \Gamma$. Hence, we have $\Gamma=\operatorname{End}_{\Lambda}^{r}(C(\Gamma))$ as above.

We shall call briefly an hereditary order an $h$-order.

Summarizing the above results, we have

THEOREM 1.7. Let $R$ be a commutative noetherian domain with field of quotients $K$. Let $\Lambda$ be an h-order over $R$ in the semi-simple K-algebra $\Sigma$. Then every order containing $\Lambda$ is also an h-order, and there is a one-to-one correspondence between two-sided idenpotent ideals $A$ in $\Lambda$ and orders $\Gamma$ containing $\Lambda$ as follows:

$$
\begin{aligned}
& \Gamma=\operatorname{End}_{\Lambda}^{r}(A), \quad A=C(\Gamma), \\
& \Gamma_{1} \supseteq \Gamma_{2} \text { if and only if } C\left(\Gamma_{1}\right) \subseteq C\left(\Gamma_{2}\right), \text { and } \\
& A_{1} \supseteq A_{2} \text { if and only if } \operatorname{End}_{\Lambda}^{r}\left(A_{1}\right) \subseteq \operatorname{End}_{\Lambda}^{r}\left(A_{2}\right) .
\end{aligned}
$$

We close this section with the following proposition:

Proposition 1.8. Let $\Lambda$ be an order in $\Sigma$, and $A$ an ideal in $\Lambda$ such that $A$ is left $\Lambda$-projective. If $\tau_{\Omega}^{l}(A)=\Omega$ for $\Omega=\operatorname{End}_{\Lambda}^{r}(A)$, then we have $\Omega \tau_{\Lambda}^{l}(A) \Omega=\Omega$.

Proof. Since $A$ is a finitely generated projective left $\Lambda$-module, we have an isomorphism $\phi: \operatorname{Hom}_{\Lambda}^{l}(A, \Lambda) \otimes_{\Lambda} \Omega \rightarrow \operatorname{Hom}_{\Omega}^{l}\left(\Omega \otimes_{\Lambda} A, \Omega\right)$ by setting $\phi(f \otimes \omega)\left(\omega^{\prime} \otimes a\right)$ $=\omega^{\prime} f(a) \omega$, where $f \in \operatorname{Hom}_{\Lambda}^{l}(A, \Lambda) ; \omega, \omega^{\prime} \in \Omega$, (since for $\lambda \in \Lambda, \phi(f \lambda \otimes \omega)\left(\omega^{\prime} \otimes a\right)$ $\left.=\omega^{\prime}(f \lambda)(a) \omega=\omega^{\prime} f(a) \lambda \omega=\phi(f \otimes \lambda \omega)\left(\omega^{\prime} \otimes a\right)\right)$. Hence, by the definition of trace ideal, we have $\tau_{\Omega}^{l}\left(\Omega \otimes_{\Lambda} A\right)=\Omega \tau_{\Lambda}^{l}(A) \Omega$. Furthermore, from the natural epimorphism: $\Omega \otimes_{\Lambda} A \rightarrow A \rightarrow 0$, we obtain that $\Omega=\tau_{\Omega}^{l}(A) \subseteq \tau_{\Omega}^{l}\left(\Omega \otimes_{\Lambda} A\right)=\Omega \tau_{\Lambda}^{l}(A) \Omega$ $\subseteq \Omega$.

Corollary 1.9. Let $\Lambda$ be an order and $A$ an idempotent ideal in $\Lambda$ which is left and right $\Lambda$-projective. If $A \neq \Lambda$, then $\operatorname{End}_{\Lambda}^{r}(A)\left(\operatorname{End}_{\Lambda}^{l}(A)\right)$ does not contain $\operatorname{End}_{\Lambda}^{l}(A)\left(\operatorname{End}_{\Lambda}^{r}(A)\right)$.

Proof. Let $\Gamma_{1}=\operatorname{End}_{\Lambda}^{r}(A)$, and $\Gamma_{2}=\operatorname{End}_{\Lambda}^{l}(A)$. We assume $\Gamma_{1} \subseteq \Gamma_{2}$. Then we have, by Lemmas 1.1 and 1.5 and Proposition 1.8, we have $\Gamma_{1}=\Gamma_{1} \tau_{\Lambda}^{l}(A) \Gamma_{1}=A \Gamma_{1}$ $\subseteq A \Gamma_{2}=A \subseteq \Lambda$. Hence, $A=\Lambda$.

2. The center of an $h$-order. The purpose of this section is to show that an $h$-order in a semi-simple algebra $\Sigma$ is the direct sum of $h$-orders in simple components of $\Sigma$, whose centers are Dedekind domains. 
Let $R$ be a commutative noetherian domain with field of quotients $K$, and let $\Lambda$ be an $h$-order in the semi-simple $K$-algebra $\Sigma$. As a preliminary to the main theorem in this section, we make the following observation.

Let $A$ be an ideal in $\Lambda$ and let $\Lambda_{1}=\operatorname{End}_{\Lambda}^{r}(A)$, and $\Lambda_{2}=\operatorname{End}_{\Lambda}^{l}(A)$. Then we have, by Lemma $1.1, \tau_{\Lambda_{1}}(A)=\Lambda_{1}$, and $\tau_{\Lambda_{2}}(A)=\Lambda_{2}$. By $A^{-1}$ we mean the subset $=\left\{x \mid \in \Sigma, x A \subseteq \Lambda_{2}\right\}=\{x \mid \in \Sigma, A x A \subseteq A\}=\left\{x \mid \in \Sigma, A x \subseteq \Lambda_{1}\right\}$. It is clear that $A^{-1}$ is left $\Lambda_{2}$ - and right $\Lambda_{1}$-ideal in $\Sigma$. Then by the definition of trace ideal, we have that $\Lambda_{1}=\tau_{\Lambda_{1}}^{l}(A)=A A^{-1}$ and $\Lambda_{2}=\tau_{\Lambda_{2}}^{r}(A)=A^{-1} A$. Consequently, we have $\Lambda_{2}=\left(A^{-1} A\right)\left(A^{-1} A\right)=A^{-1} \Lambda_{1} A$.

LEMMA 2.1. Let $\Lambda$ be an h-order in $\Sigma$, and a central element in $\Sigma$. If the ring $\Lambda[a]$ generated by $\Lambda$ and $a$ is an order, then $a$ is contained in $\Lambda$.

Proof. Let $C=C(\Lambda[a])$. Then we have, by Theorem 1.7, $\Lambda[a]=\operatorname{End}_{\Lambda}^{r}(C)$. Put $\Gamma=\operatorname{End}_{\Lambda}^{l}(C)$. By the above observation, $\Gamma=C^{-1} \Lambda[a] C$. However, since $a$ is central, $C^{-1} \Lambda[a] C \ni a$, which implies that $\Gamma \supseteq \Lambda[a]$. Hence, by Corollary 1.9, we have $C=\Lambda$, and hence, $\Lambda[a]=\Lambda$.

Proposition 2.2. Let $\Sigma=\Sigma e_{1} \oplus \Sigma e_{2} \oplus \cdots \oplus \Sigma e_{n}$ be the simple decomposition of $\Sigma$. Then for any $h-o r d e r \Lambda$, we obtain that $\Lambda=\Lambda e_{1} \oplus \cdots \oplus \Lambda e_{n}$, and that $\Lambda e_{i}$ is an h-order in $\Sigma e_{i}$ and the center of $\Lambda e_{i}$ is integrally closed over $R e_{i}$.

Proof. It is clear that $\Lambda\left[e_{i}\right]$ is a finitely generated $R$-module, and hence, $e_{i} \in \Lambda$. Therefore, we have $\Lambda=\Lambda e_{i} \oplus \cdots \oplus \Lambda e_{n}$, and $\Lambda e_{i}$ is an $h$-order over $R e_{i}$ in $\Sigma e_{i}$. The second half is also clear.

By virtue of this proposition, we may assume that $\Sigma$ is a central simple $K$-algebra. Thus, from now on we always assume that $\Sigma$ is central simple.

The essential part of the following lemma is known (cf. [2, Theorem 6.34]), but we give the proof for the sake of completeness.

Lemma 2.3. Let $\Lambda$ be an hereditary, maximal order in $\Sigma$. Then the center $R$ of $\Lambda$ is a Dedekind domain.

Proof. First, we shall show that every nonzero prime ideal $P$ in $\Lambda$ is maximal. Since $\Lambda$ is maximal, we have, by Corollary 1.9, $\tau_{\Lambda}^{l}(A)=\tau_{\Lambda}^{r}(A)=\Lambda$ and $\operatorname{End}_{\Lambda}^{r}(A)=\operatorname{End}_{\Lambda}^{l}(A)=\Lambda$ for every ideal $A$ in $\Lambda$. Hence, $\Lambda=A A^{-1}$ by the above

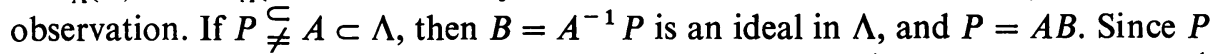
is prime and $A \underset{\neq}{\subset} P, B=A^{-1} P \subseteq P$ which implies $A^{-1} \subseteq \Lambda$ and $\Lambda=A A^{-1}$ $\subseteq A \Lambda=A$. Let $p$ be a prime ideal in $R$, and let $\Lambda_{p}=\Lambda \otimes R_{p}$. Let $M^{\prime}$ be a maximal ideal in $\Lambda_{p}$, and $M=M^{\prime} \cap \Lambda \neq(0)$. Then $M$ is prime in $\Lambda$, and hence $M$ is maximal. On the other hand, $M^{\prime}$ contains $p \Lambda_{p}$. Hence, $M \cap R=M^{\prime} \cap R_{p} \cap R=p$. Let $q$ be a prime ideal containing $p$ in $R$. Since $M$ is maximal in $\Lambda$ and $M \cap(R-q)$ $=\phi, M \otimes R_{q}$ is maximal in $\Lambda_{q}$. Hence, we have $p=M \cap R=\left(M \otimes R_{q}\right) \cap \Lambda$ $\cap R=q R_{q} \cap R=q$ as above. Therefore, the rank of $R$ does not exceed one. By Proposition 2.2, $R$ is integrally closed and hence, $R$ is a Dedekind domain. 
LEMMA 2.4. Let $R$ be a semi-local noetherian ring. Let $S$ be an $R$-algebra which is finitely generated as an $R$-module. Then for any two-sided idempotent ideal $A$ in $S$, we have $A=\bigcap_{n}(A+N)^{n}$, where $N$ is the radical of $S$. Consequently, for two-sided idempotent ideals $A$ and $B$, we have that $A \subseteq B$ if and only if $A+N \subseteq B+N$.

Proof. Let $m$ be the radical of $R$. Since $S$ is $R$-finitely generated, $N \supseteq m S$ and $N^{t} \subseteq m S$ for some integer $t$. Let $C=A+N$. Then we can easily see that $C^{n}=A+N^{n}$. Hence, $\bigcap_{n} C^{n}=\bigcap_{n}\left(A+N^{n}\right)=\bigcap_{n}\left(A+(m S)^{n}\right)$. Therefore, we have, by Artin-Rees theorem (see [11, p. 262, Theorem 9]), $A=\bigcap_{n}\left(A+(m S)^{n}\right)$.

COROllaRY 2.5. Let $\Lambda$ be an h-order in the central simple K-algebra $\Sigma$. If the center of $\Lambda$ is semi-local, then there is only a finite number of orders containing $\Lambda$ in $\Sigma$. Consequently, there exists a maximal order containing $\Lambda$ in $\Sigma$.

Proof. Since $\Lambda / N$ is semi-simple, it is clear by Theorem 1.7 and Lemma 2.4 .

THEOREM 2.6. Let $\Lambda$ be an h-order in the central simple $K$-algebra $\Sigma$. Then the center $R$ of $\Lambda$ is a Dedekind domain.

Proof. First, we assume that $R$ is local. Then there exists, by Corollary 2.5, a maximal order $\Lambda$ containing $\Gamma$. Then $\Gamma \cap K$ contains $R$ and it is a finitely generated $R$-module. However, $R$ is integrally closed by Proposition 2.2. Hence, $R$ is a Dedekind domain by Corollary 1.4 and Lemma 2.3. By the usual localization process, we have proved the theorem.

COROLlaRY 2.7. Let $\Lambda$ be an h-order over a noetherian domain $R$ in $\Sigma$. Then $\Lambda$ is $R$-projective if and only if $R$ is integrally closed in $K$.

Proof. If $R$ is integrally closed, then $R$ is a Dedekind domain. Hence, $\Lambda$ is $R$-projective by [5, p. 133, Proposition 4.2]. Conversely, let $\Lambda$ be $R$-projective. Since $\Lambda$ is a projective module over the center $Z$ of $\Lambda, Z$ is a direct summand of $\Lambda$ as $Z$-module by [4, p. 371]. Hence, $Z$ is $R$-projective. Since $Z$ and $R$ have the same quotient field $K, Z=R$.

3. Orders containing an $h$-order over a valuation ring. By virtue of Theorem 2.6, we may assume that the base ring $R$ of an $h$-order in the central simple $K$-algebra $\Sigma$ is a Dedekind domain. Thus, throughout the rest of the paper, we consider $h$-orders over a Dedekind domain $R$, unless otherwise stated.

The main purpose of this section is to give the complete description of orders containing a fixed $h$-order over a discrete rank one valuation ring.

Proposition 3.1. Let $\Lambda$ be an h-order over a Dedekind domain $R$ in $\Sigma$. Then we have: 
(1) Let $\Lambda_{2} \supseteq \Lambda_{1}$ be orders containing $\Lambda$ in $\Sigma$, then $C\left(\Lambda_{1}\right) \Lambda_{1}=\Lambda_{1}$ and $C_{\Lambda_{1}}\left(\Lambda_{2}\right)=C\left(\Lambda_{2}\right) \Lambda_{1}$.

(2) For any idempotent ideals $A$ and $B$ in $\Lambda$, we have $\operatorname{End}_{\Lambda}^{r}(A) \cap \operatorname{End}_{\Lambda}^{r}(B)$ $=\operatorname{End}_{\Lambda}^{r}(A+B)$. Furthermore, the ring $\operatorname{End}_{\Lambda}^{r}(A) \cup \operatorname{End}_{\Lambda}^{r}(B)$ generated by $\operatorname{End}_{\Lambda}^{r}(A)$ and $\operatorname{End}_{\Lambda}^{r}(B)$ in $\Lambda$ is an order if and only if $(A B)^{n}$ is idempotent for some integer $n$. In this case, we have $\operatorname{End}_{\Lambda}^{r}(A) \cup \operatorname{End}_{\Lambda}^{r}(B)=\operatorname{End}_{\Lambda}^{r}\left((A B)^{n}\right)$.

Proof. (1) By Propositions 1.6 and 1.8, we have $\Lambda_{1}=\Lambda_{1} C\left(\Lambda_{1}\right) \Lambda_{1}=C\left(\Lambda_{1}\right) \Lambda_{1}$. It is clear that $C\left(\Lambda_{2}\right) \Lambda_{1} \subseteq C_{\Lambda_{1}}\left(\Lambda_{2}\right)$ and that $C_{\Lambda_{1}}\left(\Lambda_{2}\right) C\left(\Lambda_{1}\right) \subseteq C\left(\Lambda_{2}\right)$. Hence, $C_{\Lambda_{1}}\left(\Lambda_{2}\right)=C_{\Lambda_{1}}\left(\Lambda_{2}\right) C\left(\Lambda_{1}\right) \Lambda_{1} \subseteq C\left(\Lambda_{2}\right) \Lambda_{1}$.

(2) Let $A$ and $B$ be idempotent in $\Lambda$. Since $(A+B)^{2}=A+B$, $\operatorname{End}(A) \cap \operatorname{End}(B)$ $\supseteq \operatorname{End}(A+B)$ by Theorem 1.7. However, it is clear that $\operatorname{End}(A) \cap \operatorname{End}(B)$ $\supseteq \operatorname{End}(A+B)$. Consequently, $\operatorname{End}(A) \cap \operatorname{End}(B)=\operatorname{End}(A+B)$. Let $\Gamma$ be an order containing $\operatorname{End}(A)$ and $\operatorname{End}(B)$. Then $C(\Gamma)$ is contained in $A \cap B$. Furthermore, since $C(\Gamma)$ is idempotent, $C(\Gamma)$ is contained in $(A B)^{n}$ for any $n$. Therefore, $(A B)^{t}$ is idempotent for some $t$, since $\Lambda / C(\Gamma)$ satisfies the minimal condition. Thus, we have $\Gamma \supseteq \operatorname{End}\left((A B)^{t}\right) \supseteq \operatorname{End}(A) \cup \operatorname{End}(B)$, which implies that $\operatorname{End}(A) \cup$ $\operatorname{End}(B)=\operatorname{End}\left((A B)^{t}\right)$. The converse is clear.

We shall reduce, in $\S 7$, the problems to the case where $R$ is a semi-local ring, and so we first study $h$-orders over a discrete valuation ring.

In the rest of this section, we always assume that $R$ is a discrete rank one valuation ring.

Lemma 3.2. Let $\Lambda$ be an order in $\Sigma$. Then for every ideal $A$ properly containing the radical $N$ of $\Lambda$, there exists a unique idempotent ideal $I(A)$ such that $A=I(A)+N$. Furthermore, if $A \supseteq B$ for ideals properly containing $N$, then $I(A) \supseteq I(B)$.

Proof. Let $\hat{R}$ and $\hat{\Lambda}$ be completions of $R$ and $\Lambda$ with respect to the maximal ideal in $R$, respectively. Since $R$ is a discrete, rank one valuation ring, $\hat{R}$ is a local domain of rank one. Let $\hat{K}$ be the quotient field of $\hat{R}$. Then $\hat{\Sigma}=\Sigma \otimes_{K} \hat{K}$ $\supseteq \hat{\Lambda}=\Lambda \otimes_{R} \hat{R}$, and hence, $\hat{\Lambda}$ is an order in the central simple $\hat{K}$-algebra $\hat{\Sigma}$. Furthermore, we have that $\hat{N}=N \otimes \hat{R}$ is the radical of $\hat{\Lambda}$ and that $\hat{\Lambda} / \hat{N} \approx \Lambda / N$. Let $A$ be an ideal properly containing $N$ in $\Lambda$; then $\hat{A}=A \otimes \hat{R}$ contains properly $\hat{N}$. Since $\hat{\Lambda} / \hat{N}$ is a semi-simple ring with the minimal condition, $\hat{A}$ has an element $a$ such that $a \neq 0 \bmod \hat{N}$ and $a^{2} \equiv a \bmod \hat{N}$. However, since $\hat{\Lambda}$ is a completion with respect to $\hat{N}$, we can find an idempotent element $e$ in $\hat{\Lambda}$ such that $a \equiv e \bmod \hat{N}$ by $[8$, Theorem $A]$. Since $\hat{\Lambda} e \hat{\Lambda}$ is a nonzero ideal in $\hat{\Lambda}, \hat{\Lambda} / \hat{\Lambda} e \hat{\Lambda}$ satisfies the minimal condition. Hence, $\hat{A}^{t}$ is idempotent for some integer $t$, since $\hat{A}$ contains the idempotent ideal $\hat{\Lambda} e \hat{\Lambda}$. Therefore, $A^{t}$ is idempotent by the property of completion. It is clear that $A=A^{t}+N$. The second half is an immediate consequence of Lemma 3.2.

From Theorem 1.7, Proposition 3.1 and Lemma 3.2, we have 
THEOREM 3.3. Let $R$ be a discrete, rank one valuation ring with field of quotients $K$. Let $\Lambda$ be an h-order in the central simple K-algebra $\Sigma$. Let $n$ be the number of maximal two-sided ideals in $\Lambda$. Then we have:

(1) There exist precisely $n$ maximal orders $\Lambda_{i}$ containing $\Lambda$ and $n$ minimal $\left({ }^{4}\right)$ orders $\Gamma_{i}$ containing $\Lambda$.

(2) Every order $\Omega$ properly containing $\Lambda$ is uniquely written by the form $\Omega=\bigcap_{j=1}^{r} \Lambda_{i j}=\bigcup_{k=1}^{n-r} \Gamma_{j_{k}}$, and $\Lambda=\bigcap_{i=1}^{n} \Lambda_{i}$. Consequently, the number of orders containing $\Lambda$ is equal to $2^{n}-1$, and the number of maximal two-sided ideals in $\Omega=\bigcap_{j=1}^{r} \Lambda_{i}$, is equal to $r$.

COROLlary 3.4. Maximal lengths of chain for h-orders in $\Sigma$ do not exceed the dimension of $\Sigma$ over $K$.

COROLlaRY 3.5. Let $\Lambda$ be an order as above. Then $\Lambda$ is maximal if and only if the radical of $\Lambda$ is a unique maximal two-sided ideal in $\Lambda$, and $\Lambda$ is an h-order (cf. [3, Theorem 2.3]).

If $\Lambda$ is maximal, then the radical $N$ is inversible (see [6, p. 74, Satz 9]) and hence, $N$ is $\Lambda$-projective. Thus, the corollary is true by the following result $[3$, p. 4, Theorem 2.2].

LEMMA 3.6. Let $R$ be a local noetherian ring and $\Lambda$ an $R$-algebra such that $\Lambda$ is a finitely generated $R$-module. If the radical $N$ of $\Lambda$ is a projective left $\Lambda$-module, then $\Lambda$ is hereditary. Furthermore, if the completion of $R$ with respect to the maximal ideal is an integral domain and $\Lambda$ is $R$-torsion free, then for any finitely generated left $\Lambda$-module $E$, we have that $E$ is $\Lambda$-projective if and only if $E$ is $R$-torsion free.

Proof. Let $\hat{\Lambda}, \hat{R}$ be completions of $\Lambda$ and $R$ with respect to the maximal ideal in $R$. Then by the usual argument (cf. [5, p. 129, Exercise 11]), we have $\operatorname{gl} \cdot \operatorname{dim} \Lambda \leqq \operatorname{gl} \cdot \operatorname{dim} . \hat{\Lambda} . \quad$ By $[7$, Theorem 11], gl.dim $\hat{\Lambda}=\operatorname{gl} \cdot \operatorname{dim} \hat{N}+1=1$, since $\hat{N}=N \otimes_{R} \hat{R}$ is $\hat{\Lambda}$-projective. Hence, $\Lambda$ is hereditary. Next, we assume that $\hat{R}$ is an integral domain, and that $E$ is $R$-torsion free. In order to prove that $E$ is $\Lambda$-projective, it is sufficient to show that $\hat{E}=E \otimes_{R} \hat{R}$ is $\hat{\Lambda}$-projective. Hence, we may assume that $R$ is complete. Let $0 \rightarrow K \rightarrow P \rightarrow E \rightarrow 0$ be a minimal resolution. Then $\operatorname{Tor}_{1}^{\Lambda}(\Lambda / N, E) \approx \Lambda / N \otimes_{\Lambda} K=K / N K$. Since $\Lambda$ is hereditary, $\operatorname{Tor}_{1}^{\Lambda}(\Lambda / N, E)$ is $R$-torsion free. Therefore, $K / N K=0$, which implies that $K=(0)$.

If $\Lambda$ is maximal, then for every finitely generated projective $\Lambda$-module $E$, we have $\tau_{\Lambda}(E)=\Lambda$ by [3, Proposition 3.10]. Conversely:

PROPOSITION 3.7. Let $R$ be a complete, discrete rank one valuation ring with field of quotients $K$. Let $\Lambda$ be an $h$-order in $\Sigma$. If there exists an indecomposable projective $\Lambda$-module $E$ such that $\tau_{\Lambda}(E)=\Lambda$, then $\Lambda$ is maximal.

(4) By a minimal order we mean an order $\Gamma$ containing $\Lambda$ such that if $\Gamma \supseteq \Omega \stackrel{\supsetneq}{\neq} \Lambda$ for an order $\Omega$, then $\Gamma=\Omega$. 
Proof. Since $E$ is indecomposable, $E \otimes_{R} K$ is, by [3, Proposition 2.8], isomorphic to a simple left ideal in $\Sigma=\Delta_{n}$, where $\Delta$ is the associated division ring of $\Sigma$. Hence, $\Omega=\operatorname{Hom}_{\Lambda}(E, E)$ is an order over $R$ in $\Delta$. By Lemma 3.6 and [3, Theorem A.5], $\Omega$ is hereditary. If $\Omega$ is not maximal, then there exist at least two maximal orders in $\Delta$ by Theorem 3.3 , which contradicts [3, p. 14, Corollary]. Hence, $\Lambda$ is maximal by [3, Theorem 3.6].

RemarK 1. Proposition 3.7 is not true in general unless $R$ is complete; cf. [9].

REMARK 2. By virtue of Theorem 3.3, every $h$-order is written by the intersection of a finite number of maximal orders. However, the intersection of two maximal orders, in general, is not an $h$-order. For example, let $R$ be integers and $K$ rationals. Let $\Sigma$ be a matrix ring over $K$ with degree two. A subring $\Lambda$ in $\Sigma$ which is a matrix ring over $R_{2}$ with degree two is a maximal order in $\Sigma$. We take a regular element $t_{n}=1 / 2^{n} e_{1,1}+e_{2,1}+2^{n} e_{2,2}$, where the $e_{i, j}^{1}$ 's are matrix units. Then $\Omega \cap t_{n} \Omega t_{n}^{-1}=R_{2} f_{1,1}+2^{2 n} R_{2} f_{1,2}+R f_{2,1}+R f_{2,2}$, where $f_{i, j}$ $=t_{n} e_{i, j} t_{n}^{-1}$. There exists an order $R_{2} f_{1,1}+2 R_{2} f_{1,2}+R_{2} f_{2,1}+R_{2} f_{2,2}$ between $t_{n} \Omega t_{n}^{-1}$ and $\Omega \cap t_{n} \Omega t_{n}^{-1}$, and hence, $\Omega \cap t_{n} \Omega t_{n}^{-1}$ is not hereditary for any $n \geqq 1$.

4. Relations between $h$-orders over a valuation ring. Let $R$ be a Dedekind domain with field of quotients $K$ and $\Sigma$ the central simple $K$-algebra. For two orders $\Lambda_{1}$ and $\Lambda_{2}$ in $\Sigma$, we say that $\Lambda_{1}$ and $\Lambda_{2}$ belong to the same type through $C$ if there exists a left $\Lambda_{1}$ - and right $\Lambda_{2}$-ideal $C$ in $\Sigma$ such that End ${ }_{\Lambda_{1}}^{l}(C)=\Lambda_{2}$ and End $_{\Lambda_{2}}^{r}(C)=\Lambda_{1}$ (notation $\left(\Lambda_{1}, C, \Lambda_{2}\right)$ ); cf. [1]. It is clear that two maximal orders belong to the same type through the conductor.

Furthermore, if $\Lambda_{1}$ and $\Lambda_{2}$ are $h$-orders, then we have by Lemma 1.1 that $\Lambda_{1}=\tau_{\Lambda_{1}}^{l}(C)=C C^{-1}$ and $\Lambda_{2}=\tau_{\Lambda_{2}}^{r}(C)=C^{-1} C$ and hence, $\Lambda_{2}=C^{-1} \Lambda_{1} C$.

It is clear that in the category of $h$-orders the relation of the same type is reflexible and transitive.

Proposition 4.1. Let $R$ be a Dedekind domain and let $\Lambda_{1}$ and $\Lambda_{2}$ belong to the same type. If $\Lambda_{1}$ is maximal, then $\Lambda_{2}$ is maximal. If $\Lambda_{1}$ and $\Lambda_{2}$ are $h$ orders, then there is a one-to-one correspondence between ideals $A_{1}$ in $\Lambda_{1}$ and ideals $A_{2}$ in $\Lambda_{2}$ by the mapping $A_{1} \rightarrow C^{-1} A_{1} C$ and $A_{2} \rightarrow C A_{2} C^{-1}$ which preserves inclusion and multiplication of ideals (cf. [3, Theorem A.5; 6, p. 75, Satz 12]).

Proof. The second half is clear from the above observation. By [3, p. 2, Corollary and Lemma 2.4], we may restrict ourselves to the case where $R$ is a local ring. Since $\Lambda_{1}$ is maximal, $\tau_{\Lambda_{1}}(C)=\Lambda_{1}$ by [3, Proposition 3.10]. Hence, $\Lambda_{2}$ is maximal by Corollary 3.5 and [3, Theorem A.5].

Proposition 4.2. Let $\Lambda$ be an h-order over a Dedekind domain $R$ in $\Sigma$. Let $\Omega_{i}$ be orders containing $\Lambda(i=1,2, \cdots, t)$. If $\left(\Omega_{1}, C_{1}, \Omega_{2}\right)\left(\Omega_{2}, C_{2}, \Omega_{3}\right), \cdots,\left(\Omega_{t}, C_{t}, \Omega_{1}\right)$ belong to the same type, then there is no order in $\Sigma$ containing all the $\Omega_{i}$ 's, where $C_{i}=C_{\Lambda}\left(\Omega_{i}\right)$. 
Proof. We assume that there exists an order $\Gamma$ containing all the $\Omega_{i}$ 's. Then $C(\Gamma) \subseteq \bigcap_{i} C_{i}$. Since $C(\Gamma)$ is idempotent, there exists an integer $r$ such that $B=\left(C_{1} C_{2} \cdots C_{t}\right)^{r}$ is idempotent. Then we have, by Proposition 3.1, $\Omega_{1}$ $\cup \Omega_{2} \cup \cdots \cup \Omega_{t}=\operatorname{End}_{\Lambda}^{r}(B)$. On the other hand, by interchanging left and right, we obtain by Proposition 1.6 that $\Omega_{2} \cup \Omega_{3} \cup \ldots \cup \Omega_{t} \cup \Omega_{1}=$ End $_{\Lambda}^{l}(B)$ as above, which is a contradiction to Corollary 1.9.

THEOREM 4.3. Let $R$ be a discrete, rank one valuation ring with field of quotients $K$. Let $\Lambda$ be an h-order in $\Sigma$. Then two orders $\Omega_{1}$ and $\Omega_{2}$ containing $\Lambda$ belong to the same type if and only if $\Omega_{1}$ and $\Omega_{2}$ have the same number of maximal ideals.

Proof. If $\Omega_{1}$ and $\Omega_{2}$ belong to the same type, then they have the same number of maximal ideals by Proposition 4.1. Conversely, if $\Omega_{1}$ and $\Omega_{2}$ have the same number of maximal ideals, then $\Omega_{1}=\bigcup_{j=1}^{r} \Gamma_{i j}$ and $\Omega_{2}=\bigcup_{i=1}^{r} \Gamma_{k_{l}}$ by Theorem 3.3 , where the $\Gamma$ 's are minimal orders containing $\Lambda$. First we shall show that every minimal order belongs to the same type. Let $\left\{\Gamma_{1}, \cdots, \Gamma_{n}\right\}$ be the set of minimal orders containing $\Lambda$. Let $C_{1}=C\left(\Gamma_{1}\right)$; then we have, by Theorem 1.7, that $\Gamma_{1}=\operatorname{End}_{\Lambda}^{r}\left(C_{1}\right)$. Furthermore, $\operatorname{End}_{\Lambda}^{l}\left(C_{1}\right)$ is a minimal order by Proposition 4.1, say $\Gamma_{2}=\operatorname{End}_{\Lambda}^{l}\left(C_{1}\right)$. Then $\Gamma_{1}$ and $\Gamma_{2}$ belong to the same type. Again from the conductor $C_{2}=C\left(\Gamma_{2}\right)$, we obtain the same type $\left(\Gamma_{2}, C_{2}, \Gamma_{3}\right)$. Repeating this argument, we may have a set of minimal orders $\Gamma_{i}$ such that $\left(\Gamma_{1}, C_{1}, \Gamma_{2}\right)$, $\left(\Gamma_{2}, C_{2}, \Gamma_{3}\right), \cdots,\left(\Gamma_{t}, C_{t}, \Gamma_{1}\right)$. If $t<n$, then $\bigcup_{i=1}^{t} \Gamma_{i}$ is an order in $\Sigma$ by Theorem 3.3. Therefore, $t=n$ by Proposition 4.2. Thus we have proved the theorem for $r=1$. We assume that $r \geqq 2$, and $\Gamma=\bigcup_{j=1}^{t} \Gamma_{i_{j}}=\bigcup_{l=1}^{t} \Gamma_{k_{l}}, t<r$ and that $\Gamma_{i_{p}} \neq \Gamma_{k_{g}}$ if $p, q>t$. We consider $\Omega=\Gamma \cup \Gamma_{k_{t+1}} \cup \Gamma_{i_{t+2}} \cup \cdots \cup \Gamma_{i_{r}}$. Then $\Omega_{1}$ and $\Omega$ are minimal orders containing $\Gamma \cup \Gamma_{i_{t+2}} \cup \cdots \cup \Gamma_{i_{r}}$. Hence, they belong to the same type of the above argument. Therefore, by using the induction on $t$, we have proved that $\Omega_{1}$ and $\Omega_{2}$ belong to the same type.

In the rest of this section, we shall consider associated division ring of simple components of $\Lambda / N$, where $N$ is the radical of $\Lambda$.

Proposition 4.4. Let $\Omega_{1}$ and $\Omega_{2}$ be h-orders with radicals $N_{1}$ and $N_{2}$ respectively, which belong to the same type through $A$. Then we have an isomorphism $\Omega_{1} / N_{1}$ to $\operatorname{Hom}_{\Omega_{2} / N_{2}}^{r}\left(A / A N_{2}, A / A N_{2}\right)$ by the natural mapping. The associated division rings of $\Omega_{1} / N_{1}$ and $\Omega_{2} / N_{2}$ are isomorphic.

Proof. Let $m$ be the maximal ideal in $R$. Since $A$ is $\Omega_{2}$-projective, we have $\Omega_{1} / m \Omega_{1}=\operatorname{Hom}_{\Omega_{2}}^{r}(A, A) \otimes_{R} R / m=\operatorname{Hom}_{\Omega_{2} / m \Omega_{2}}^{r}(A / m A, A / m A) . \bar{\Omega}_{i}=\Omega_{i} / m \Omega_{i}(i=1,2)$ are semi-primary rings with radical $N_{i} / m \Omega_{i}$ and $\bar{A}=A / m A$ is $\bar{\Omega}_{2}$-projective. Let $\bar{\Omega}_{2}=\sum_{i=1}^{n} \sum_{j=1}^{p(i)} e_{i, j} \bar{\Omega}_{2}$ be a decomposition of indecomposable components. Then by [7, Corollary 4], $A=\sum_{i=1}^{m} \sum_{j=1}^{n(i)} e_{i, j} \overline{\Omega_{2}}$. It is clear that the images of elements in $\operatorname{Hom}_{\bar{\Omega}_{2}}^{r}\left(A, \bar{\Omega}_{2}\right)$ are contained in $\sum_{i=1}^{m} e_{i, j} \bar{\Omega}_{2}+\sum_{l=m+1}^{n} e_{l, k} \bar{N}_{2}$. However, $\tau_{\Omega_{2}}^{r}(A)=\Omega_{2}$ implies $\tau_{\bar{\Omega}_{2}}^{r}(\bar{A})=\bar{\Omega}_{2}$. Hence, we have $n=m$. Furthermore, we have 
a natural homomorphism $\phi$ of $\operatorname{Hom}_{\bar{\Omega}_{2}}^{r}(\bar{A}, \bar{A})$ to $\operatorname{Hom}_{\mathbf{\Omega}_{2} / N_{2}}\left(\bar{A} / \bar{A} N_{2}, \bar{A} / \bar{A} N_{2}\right)$. Since $\bar{A} / \bar{A} N_{2}=\sum_{i=1}^{n} \sum_{j=1}^{n(i)} e_{i, j} \Omega_{2} / N_{2}$, we can easily see that $\phi$ is epimorphic. On the other hand, since $\operatorname{Hom}_{\Omega_{2} / N_{2}}^{r}\left(A / A N_{2}, A / A N_{2}\right)$ is semi-simple, we have $\phi^{-1}(0) \supseteq \bar{N}_{1}$. Therefore, from the facts that $n=m$ and that the number of simple components of $\Omega_{1} / N_{1}$ and $\Omega_{2} / N_{2}$ are same by Proposition 4.1, we have $\phi^{-1}(0)=\bar{N}_{1}$.

CoROllary 4.5. Let $\Lambda_{1}$ and $\Lambda_{2}$ be h-orders such that every simple components of $\Lambda_{i} / N_{i}$ is a matrix ring with same degree $n$ (cf. [9]). Then $\Lambda_{1}$ and $\Lambda_{2}$ belong to the same type if and only if $\Lambda_{1}$ and $\Lambda_{2}$ are isomorphic by an innerautomorphism. For any ideal $A$ in $\Lambda_{1}$, we have that $\tau_{\Lambda_{1}}^{l}(A)=\Lambda_{1}$ if and only if $A$ is principal as a left (right) $\Lambda_{1}$-module. Consequently, every maximal order is isomorphic by an inner-automorphism, and every one-sided ideal is principal (cf. [3, Proposition 3.5]).

Proof. If $\Lambda_{1}=\alpha \Lambda_{2} \alpha^{-1}$ for some element $\alpha$ in $\Sigma$, then $\Lambda_{1}$ and $\Lambda_{2}$ belong to the same type through $\Lambda_{1} \alpha=\alpha \Lambda_{2}$. Conversely, we assume that $\Lambda_{1}$ and $\Lambda_{2}$ belong to the same type through $A$. Then $\Lambda_{1} / N_{1} \approx \operatorname{Hom}_{\Lambda_{2} / N_{2}}^{r}\left(A / A N_{2}, A / A N_{2}\right)$ by the proposition. Let $A / m A=\sum_{i=1}^{r} \sum_{j=1}^{p(i)} e_{i j} \Lambda_{2} / \Lambda_{2} m$; then $\Lambda_{1} / N_{1} \approx \sum_{i=1}^{r} \otimes\left(\Delta_{i}\right)_{p(i)}$. Hence, by assumption, we have $p(i)=n$ for all $i$. Therefore, $A / m A$ is isomorphic to $\Lambda_{2} / m \Lambda_{2}$ as a right $\Lambda_{2}$-module, which implies that $A$ is a principal ideal, namely $A=\alpha \Lambda_{2}$. Thus, $\Lambda_{1}=\operatorname{Hom}_{\Lambda_{2}}\left(\alpha \Lambda_{2}, \alpha \Lambda_{2}\right)=\alpha \Lambda_{2} \alpha^{-1}$. Let $B$ be an ideal in $\Lambda_{1}$. If $\tau_{\Lambda_{1}}^{l}(B)=\Lambda_{1}$, then $\operatorname{End}_{\Lambda_{1}}^{l}(B)$ and $\Lambda_{1}$ belong to the same type by Lemma 1.1. Therefore, End $\Lambda_{1}^{l}(B)=\Lambda_{1}$, and $B$ is principal. Let $\Omega_{1}$ and $\Omega_{2}$ be maximal orders and $C=C_{\Omega_{2}}\left(\Omega_{1}\right)$. Since $\left[\Omega_{1} / m \Omega_{1}: R / m\right]=\left[\Omega_{2} / m \Omega_{2}: R / m\right]=[\Sigma: K]$, and $\Omega_{1} / N_{1} \approx \operatorname{Hom}_{\Omega_{2} / N_{2}}^{r}\left(C / C N_{2}, C / C N_{2}\right), \Omega_{1} / N_{1} \approx \Delta_{n} \approx \Omega_{2} / N_{2}$.

THEOREM 4.6. For any h-order $\Lambda$ in $\Sigma$, the associated division rings of simple components of $\Lambda / R(\Lambda)$ are isomorphic to a division ring which does not depend on $\Lambda$. Let $\Omega \supseteq \Lambda$ be h-orders such that $\Omega / R(\Omega) \approx \sum_{i=1}^{s} \oplus \Delta_{n(i)}$ and $\Lambda / R(\Lambda)$ $\approx \sum_{i=1}^{t} \oplus \Delta_{m(i)}$. Then there is a one-to-one mapping $\pi$ of $\{1,2, \cdots, s\}$ into $\{1,2, \cdots, t\}$ such that $n(i) \geqq m(\pi(i))$ and this inequality is not equal for some $j$, where $R($ ) means the radical of ring.

Proof. We use the same notations as in the proof of Proposition 4.4. Let $C=C(\Omega)$ and $\Omega / R(\Omega) \approx\left(\Delta_{1}(\Omega)\right)_{n(1)} \oplus \cdots+\left(\Delta_{s}(\Omega)\right)_{n(s)}$, and $\Lambda / R(\Lambda) \approx\left(\Delta_{1}(\Lambda)\right)_{m(1)}$ $+\cdots \oplus\left(\Delta_{s}(\Lambda)\right)_{m(s)} \oplus \cdots \oplus\left(\Delta_{t}(\Lambda)\right)_{m(t)} . \quad \bar{C}=\sum_{i=1}^{s^{\prime}} \sum_{j=1}^{p(i)} e_{\alpha(i), j} \bar{\Lambda}$ and $\bar{C} / \bar{C} N$ $=\sum_{i=1}^{s^{\prime}} \sum_{j=1}^{p(i)} e_{\alpha(i), j} \Lambda / N$, where $N=R(\Lambda)$. Then we have a natural epimorphism $\phi$ of $\bar{\Omega}=\operatorname{Hom}_{\bar{\Lambda}}^{r}(\bar{C}, \bar{C})$ to $\operatorname{Hom}_{\bar{\Lambda} / \bar{N}}^{r}(\bar{C} / \bar{C} N, \bar{C} / \bar{C} N)$ and $\phi^{-1}(0) \supseteq R(\bar{\Omega})$ (cf. the proof of Proposition 4.5). Since $C / C N$ is $\Lambda / N$-module, we have $\sum_{l=1}^{s^{\prime}} \sum_{j=1}^{p(i)} e_{\alpha(i), j} \Lambda / N$ $=C+N / N \oplus C \cap N / C N=\sum_{i=1}^{s} \sum_{j=1}^{m(i)} e_{i, j} \Lambda / N \oplus C \cap N / C N$ by Theorem 3.3, where we assume that $C+N / N \approx \sum_{i=1}^{s} \oplus\left(\Delta_{i}(\Lambda)\right)_{m(i)}$. Hence, $s^{\prime} \geqq s$. On the other hand, $\operatorname{Hom}_{\Lambda / N}^{r}(C / C N, C / C N)$ has $s^{\prime}$ simple components, and hence we obtain that $s=s^{\prime}$ and $\phi^{-1}(0)=R(\bar{\Omega})$. Therefore, $n(i) \geqq m(\pi(i))$ and $\Delta_{i}(\Omega) \approx \Delta_{\pi(i)}(\Lambda)$. 
By Theorem 3.3, each simple component $\left(\Delta_{\iota}(\Lambda)\right)_{m(i)}$ of $\Lambda / N$ corresponds to a maximal order $\Omega$ containing $\Lambda$ such that $(C(\Omega)+N) / N \approx\left(\Delta_{i}(\Lambda)\right)_{m(i)}$. Hence, every associated division ring $\Delta_{i}(\Lambda)$ is isomorphic to that of $\Omega / R(\Omega)$, which does not depend on $\Lambda$ by Corollary 4.5. Finally, if we show that $C \cap N / C N \neq(0)$ for $\Omega \neq \Lambda$, then we complete the proof. From an exact sequence: $0 \rightarrow C \rightarrow \Lambda \rightarrow \Lambda / C \rightarrow 0$, we have $\operatorname{Tor}_{\Lambda}^{l}(\Lambda, \Lambda / N) \rightarrow \operatorname{Tor}_{\Lambda}^{l}(\Lambda / C, \Lambda / N) \rightarrow C \otimes_{\Lambda} \Lambda / N \rightarrow \Lambda / N$. Hence, $C \cap N / C N$ $\approx \operatorname{Tor}_{\Lambda}^{l}(\Lambda / C, \Lambda / N)$. If $\operatorname{Tor}_{\Lambda}^{l}(\Lambda / C, \Lambda / N)=0$, then $\operatorname{Tor}_{\hat{\Lambda}}^{l}(\hat{\Lambda} / \hat{C}, \hat{\Lambda} / \hat{C})=0$ by the usual argument, where $\hat{\Lambda}$ means a completion with respect to the maximal ideal of $R$. Hence, $\hat{\Lambda} / \hat{C}$ is $\hat{\Lambda}$-projective by [7, Theorem 11]. Therefore, we have $\hat{C}=\hat{\Lambda}$ which implies $C=\Lambda$.

5. Criteria of $h$-orders. In this section we shall show the converse of Theorem 3.3.

Lemma 5.1. Let $\Lambda$ be an order in $\Sigma$ and $\Omega$ a maximal order containing $\Lambda$. If $\Omega$ is left $\Lambda$-projective, then $C(\Omega)$ is a minimal idempotent ideal in $\Lambda$.

Proof. $C=C(\Omega)$ is left $\Omega$-projective, and hence, $\Lambda$-projective. By Proposition 1.6, $\tau_{\Lambda}^{l}(C)=C$. Hence, $C$ is idempotent by Lemma 1.5. Let $C_{0}$ be an idempotent ideal contained in $C$. Then $\operatorname{End}_{\Lambda}^{r}\left(C_{0}\right)=\Omega$. Therefore, $C_{0}$ is left $\Lambda$ projective. Thus, $C_{0}=C\left(\operatorname{End}^{r}\left(C_{0}\right)\right)=C$ by Theorem 1.7.

Lemma 5.2. Let $C$ be a maximal idempotent ideal in an order $\Lambda$ such that $\operatorname{End}_{\Lambda}^{r}(C) \neq \Lambda$. Then $\operatorname{End}_{\Lambda}^{r}(M) \neq \Lambda$ for the maximal ideal $M$ containing $C$.

Proof. Let $\Omega_{1}=\operatorname{End}_{\Lambda}^{r}(M)$ and $\Omega=\operatorname{End}_{\Lambda}^{r}(C)$. We have, by Lemma 3.2 , $M^{n}=C$ for some $n$. We assume $\Omega_{1}=\Lambda$. We consider the following two cases: (1) $\tau_{\Lambda}^{r}(M)=\Lambda$, (2) $\tau_{\Lambda}^{r}(M)=M$.

CASE 1. If $\tau_{\Lambda}^{l}(M)=\Lambda$, then we have $M M_{r}^{-1}=M_{l}^{-1} M=\Lambda$, where $M_{r}^{-1}$ $=\{x \mid \in \Sigma, M x \subseteq \Lambda\}$, and $M_{l}^{-1}=\{x \mid \in \Sigma, x M \subseteq \Lambda\}$. Hence, $M$ is inversible, which implies that $C$ is also inversible. However, $C$ is an idempotent ideal $\neq \Lambda$. Therefore, $\tau_{\Lambda}^{l}(M) \neq \Lambda$, and hence, $\tau_{\Lambda}^{l}(M)=M$. By assumptions $\Omega_{1}=\Lambda$ and $\tau_{\Lambda}^{r}(M)=M$, $M$ is left $\Lambda$-projective. Hence, $M=\tau_{\Lambda}^{l}(M) M=M^{2}$ by Lemma 1.1. Thus $M=C$, which is a contradiction to $\Omega \neq \Lambda$.

CASE 2. Since $M^{n}=C$, there exists an integer $i \geqq 2$ such that $M^{i-1}=C$, and $M^{i}=C . \Lambda \supseteq \Omega C=\Omega M^{i-1} M$. Since $\tau_{\Lambda}^{r}(M)=M$, we have $\Omega M^{i-1} M \subseteq M$. Hence, $\Omega M^{i-1} \subseteq \operatorname{End}_{\Lambda}^{r}(M)=\Lambda$, which implies $M^{i-1} \subseteq C$. Thus, we know $\Omega_{1} \neq \Lambda$.

THEOREM 5.3. Let $R$ be a discrete rank one valuation ring with field of quotients $K$. Let $\Lambda$ be an order over $R$ in the central simple $K$-algebra $\Sigma$, such that $\Lambda / N$ has $n$ simple components. We assume that every maximal order $(\supseteq \Lambda)$ is left $\Lambda$-projective. If there exists a maximal chain of orders $\Delta_{i}$ containing $\Lambda\left(\Delta_{1} \supset \Delta_{2} \supset \cdots \supset \Delta_{n}=\Lambda\right)$ such that every $\Delta_{i}$ is left $\Lambda$-projective and $\Delta_{i}$ has 
precisely $i$ maximal two-sided ideals, then $\Lambda$ is hereditary, and the radical of $\Lambda$ is inversible.

Proof. We shall prove the theorem by induction on $n$. If $n=1$, then $\Lambda$ is maximal and hence, $\Lambda$ is hereditary. We assume that the theorem is true for order with $n-1$ maximal ideals. Let $\Lambda$ be an order as in the theorem. Then $\Delta_{n-1}$ satisfies the conditions in the theorem by Lemma 1.3. Hence, $\Delta_{n-1}$ is hereditary. We denote $\Delta_{n-1}$ by $\Gamma_{1}$. Let $\left\{\Omega_{1}, \Omega_{2}, \cdots, \Omega_{n-1}\right\}$ be the set of maximal orders containing $\Gamma_{1}$ and $D_{i}=C_{\Lambda}\left(\Omega_{i}\right)$. Then the $D_{i}$ 's are minimal idempotent and left $\Lambda$-projective. Let $C_{1}=C\left(\Gamma_{1}\right)$; then $C_{1}=\sum_{i=1}^{n-1} D_{i}$, and $\Gamma_{1}=\operatorname{End}_{\Lambda}^{r}\left(C_{1}\right)$ by Proposition 1.6. Furthermore, $C_{1} \Gamma_{1}$ is idempotent in $\Gamma_{1}$ and $\operatorname{End}_{\Gamma_{1}}^{r}\left(C_{1} \Gamma_{1}\right) \subseteq \bigcap{ }_{i} \operatorname{End}_{\Gamma_{1}}^{r}\left(D_{i} \Gamma_{1}\right)=\bigcap_{i} \Omega_{i}=\Gamma_{1}$. Since $\Gamma_{1}$ is hereditary, $C_{1} \Gamma_{1}=\Gamma_{1}$. Therefore, $\tau_{\Gamma_{1}}^{l}\left(C_{1}\right)=\Gamma_{1}$. Let $\Gamma_{2}=\operatorname{End}_{\Lambda}^{l}(C)$. Then $\Gamma_{2}$ is also hereditary by $[3$, Theorem A.5], and $\Gamma_{2}$ has $n-1$ maximal ideals. Let $\left\{\Omega_{1}^{\prime}, \Omega_{2}^{\prime}, \cdots, \Omega_{n-1}^{\prime}\right\}$ be the set of maximal orders containing $\Gamma_{2}$ and $D_{i}^{\prime}=C\left(\Omega_{i}^{\prime}\right)$. Since $\Lambda / N$ has $n$ simple components and the $D_{i}$ 's and the $D_{i}^{\prime}$ 's are minimal idempotent in $\Lambda$, we may assume that $\Omega_{i}=\Omega_{i}^{\prime}$ for $i \leqq n-2$ and $\Omega_{1}, \Omega_{2}, \cdots, \Omega_{n-1}, \Omega_{n}=\Omega^{\prime}{ }_{n-1}$ are the set of maximal orders containing $\Lambda$. Since $C_{2}^{\prime}=C\left(\Gamma_{2}\right) \supseteq D_{1}+D_{2}+\cdots+D_{n-2}$ $+D_{n}, C_{2}=I\left(C_{2}^{\prime}\right)+N=D_{1}+\cdots+D_{n-2}+D_{n}$ by Lemma 3.2. Furthermore, $\Gamma^{\prime}=\operatorname{End}_{\Lambda}^{r}\left(C_{2}\right) \supseteq \operatorname{End}_{\Lambda}^{r}\left(C_{2}^{\prime}\right) \supseteq \Gamma_{2}$ and $\Gamma^{\prime} \subseteq \bigcap_{i \neq n-1} \Omega_{i}=\Gamma_{2}$. Repeating this argument, we have the following set of $h$-orders $\Gamma_{i}:\left(\Gamma_{1}, C_{1}, \Gamma_{2}\right),\left(\Gamma_{2}, C_{2}, \Gamma_{3}\right), \cdots$, $\left(\Gamma_{i}, C_{i}, \Gamma_{i+1}\right)$ and the $C_{i}$ 's are maximal idempotent ideals. Let $D\left(\Gamma_{j}\right)$ be the left conductor of $\Gamma_{j}$; then $D\left(\Gamma_{j}\right) \supset C_{j-1}$. Hence, $I\left(D\left(\Gamma_{j}\right)\right)=C_{j-1}$. If $\Gamma_{i+1}=\Gamma_{j+1}$ for $j<i$, then $C_{j}=I\left(D\left(\Gamma_{j+1}\right)\right)=I\left(D\left(\Gamma_{i}\right)\right)=C_{i}$, and hence, $\Gamma_{j}=\operatorname{End}_{\Lambda}^{r}\left(C_{j}\right)=\operatorname{End}_{\Lambda}^{r}\left(C_{i}\right)$ $=\Gamma_{i}$. Therefore, we assume $\Gamma_{i+1}=\Gamma_{1}$. By using the same argument as the proof of Theorem 4.3, we shall show that $i=n$. If $i<n$, there exists a maximal order $\Omega$ containing all the $\Gamma_{i}$ 's by the construction of $\Gamma_{i}$. Hence, $C(\Omega)$ is contained in the idempotent ideal $B=I\left(C_{1} \cdots C_{i}\right)$. Let $\Delta=\operatorname{End}_{\Lambda}^{r}(B)$; then $\Delta \supset \Gamma_{1} \cup \Gamma_{2}$ $\cup \cdots \cup \Gamma_{i}$. Since $\Gamma_{1}$ is left $\Lambda$-projective and hereditary, there is a one-to-one correspondence between orders $\Delta^{\prime}$ containing $\Gamma_{1}$ and idempotent ideals contained in $C$. Hence, we have $\operatorname{End}_{\Lambda}^{r}(B)=\Gamma_{1} \cup \Gamma_{2} \cup \cdots \cup \Gamma_{1}$. It is clear that

$$
\operatorname{End}_{\Lambda}^{l}(B) \supset \Gamma_{2} \cup \Gamma_{3} \cdots \cup \Gamma_{i} \cup \Gamma_{1} .
$$

On the other hand, $B$ is right $\Lambda$-projective by Proposition 1.6. Hence, we have a contradiction to Corollary 1.9. Thus, we have proved that for every maximal idempotent ideal $C_{i}, \operatorname{End}_{\Lambda}^{r}\left(C_{i}\right) \neq \Lambda \neq \operatorname{End}_{\Lambda}^{l}\left(C_{\imath}\right)$. Let $M_{j}$ be a maximal ideal in $\Lambda$ containing $C_{j}$ and $C_{j}=I\left(M_{j}\right)=M_{j}^{t}$. Then $\operatorname{End}_{\Lambda}^{r}\left(M_{j}\right)=\Gamma_{j}$ and $\operatorname{End}_{\Lambda}^{l}\left(M_{j}\right)=\Gamma_{j+1}$ by Lemma 5.2. Since $C_{j} \Gamma_{j}=\Gamma_{j}, M_{j} \Gamma_{j}=\Gamma_{j}$. Let $N$ be the radical of $\Lambda$. Then $N=\bigcap_{j} M_{j}$ and $\Lambda \supset M_{j-1} \Gamma_{j} \supseteq N \Gamma_{j} \supseteq M_{p_{1}} M_{p_{2}} \cdots M_{p_{n-2}} M_{j-1} M_{j} \Gamma_{j}=M_{p_{1}} M_{p_{2}}$ $\cdots M_{p_{n-2}} M_{j-1}$. Therefore, $\tau_{\Lambda}^{l}(N) \supseteq \sum_{i} M_{1} \cdots M_{i-1} M_{i+1} \cdots M_{n}+N=\Lambda$. Similarly, we have $\tau_{\Lambda}^{r}(N)=\Lambda$. Therefore, $N$ is inversible, and hence, $\Lambda$ is hereditary by Lemma 3.6 . 
COROLlary 5.4. Let $\Lambda$ be an order such that $\Lambda$ contains precisely two maximal ideals. If every maximal order containing $\Lambda$ is left $\Lambda$-projective, then $\Lambda$ is hereditary.

Proof. Let $\Omega$ be a maximal order containing $\Lambda$. Then there are no orders between $\Omega$ and $\Lambda$, and hence, $\Lambda$ is hereditary by the theorem.

In the contrast with Lemma 3.6, we have

CoRollary 5.5. If every (fractional) idempotent ideal with respect to $\Lambda$ is left $\Lambda$-projective, then $\Lambda$ is hereditary.

Proof. We assume that $\Lambda$ has $n$ maximal ideals. Then first we shall show that there exist precisely $n$ maximal orders $\Omega_{i}$ containing $\Lambda$ and $\Lambda=\bigcap \Omega_{i}$. Let $\left\{\Omega_{1}, \Omega_{2}, \cdots, \Omega_{r}\right\}$ be the set of maximal orders containing $\Lambda$, and $C_{i}=C(\Omega)$. Since the $C_{i}$ 's are minimal idempotent, $r \leqq n$. If $r<n$, there exists a minimal idempotent ideal $C \neq C_{i}$ for all $i \leqq r$. Then $\operatorname{End}_{\Lambda}^{l}(C)$ is contained in some $\Omega_{\pi(i)}=\operatorname{End}_{\Lambda}^{l}\left(C_{i}\right)$. Hence, $C \supseteq D\left(\operatorname{End}_{\Lambda}^{l}\left(C_{i}\right)\right)=\tau_{\Lambda}^{r}\left(C_{i}\right)=C_{i}$ by Proposition 1.6. It is clear that $C\left(\bigcap \Omega_{i}\right) \supseteq \sum C_{i}=\Lambda$, and hence, $\Lambda=\bigcap \Omega_{i}$. Let $D=\sum_{j=1}^{n-1} C_{j}$; then since $D$ is left $\Lambda$-projective, $\Lambda \neq \operatorname{End}_{\Lambda}^{l}(D) \subset \bigcap_{j \neq n} \operatorname{End}^{1}\left(C_{j}\right)$. Therefore, we can prove the corollary by induction on $n$ with Theorem 5.3.

6. Two-sided ideals with respect to an $h$-order. In this section, we shall study a group structure of the set of two-sided (fractional) ideals with respect to an $h$-order $\Lambda$.

For this purpose, we quote the following definition (cf. [7, p. 76]):

Definition. For two-sided ideals $A, B$ the product $A B$ is called a characteristic product, if $A^{\prime} \supseteq A, B^{\prime} \supseteq B$ and $A B=A^{\prime} B^{\prime}$; then $A^{\prime}=A$ and $B^{\prime}=B$ for any ideals $A^{\prime}$ and $B^{\prime}$.

Let $A$ be an ideal with respect to an $h$-order $\Lambda$. Then $\Omega_{1}=\operatorname{End}_{\Lambda}^{r}(A)$ and $\Omega_{2}=\operatorname{End}_{\Lambda}^{l}(A)$ are $h$-orders containing $\Lambda$, and $A A^{-1}=\Omega_{1}, A^{-1} A=\Omega_{2}$. Let $B$ be another ideal. If $\Omega_{2}=\operatorname{End}_{\Lambda}^{l}(B)$, then $A B$ is a characteristic product. Because, if $A^{\prime} \supseteq A$ and $B^{\prime} \supseteq B$ and $A^{\prime} B^{\prime}=A B$, then $A B \subseteq A^{\prime} B \subseteq A^{\prime} B^{\prime}$ and hence, $A B=A^{\prime} B$. Therefore, $A^{\prime}=A^{\prime} B B^{-1}=A \Omega_{2}=A$. Conversely, if $A B$ is characteristic, then $A \Omega_{2} B=A B$ and hence, $\Omega_{2} B=B$, which implies $\Omega_{2} \subseteq \operatorname{End}_{\Lambda}^{r}(B)$. Similarly, we have $\Omega_{2} \supseteq \operatorname{End}_{\Lambda}^{r}(B)$ (cf. [2, p. 182, Theorem 4.51]).

Now, let $\Lambda$ be an $h$-order over a discrete, rank one valuation ring, which has $n$-maximal two-sided ideals in $\Lambda$. Let $\Omega_{i}^{n-j}(j=0,1, \cdots, n-1 ; i=1,2), \cdots,\left(\begin{array}{c}n \\ j\end{array}\right)$ be the set of orders containing $\Lambda$, and $\Omega_{i}^{n-j}$ has $n-j$ maximal two-sided ideals in $\Omega_{i}^{n-j}$. By $G_{l, m}^{n-j}$ we denote the set of two-sided fractional ideals $A$ with respect to $\Lambda$ such that $\operatorname{End}_{\Lambda}^{r}(A)=\Omega_{l}^{n-j}$, and $\operatorname{End}_{\Lambda}^{l}(A)=\Omega_{m}^{n-j}$. Then for $A \in G_{l, m}^{n-j}$, $B \in G_{p, q}^{n-t}$ we have the characteristic product $A B \in G_{l, q}^{n-j}$ if $t=j$ and $m=p$; if not then $A B$ is not characteristic. Let $A$ be an ideal with respect to $\Lambda$. Since $\operatorname{End}_{\Lambda}^{r}(A)$ and $\operatorname{End}_{\Lambda}^{l}(A)$ belong to the same type and hence, $A$ belongs to some $G_{l, m}^{n-j}$. Conversely, since $\Omega_{l}^{n-j}$ and $\Omega_{m}^{n-j}$ belong to the same type, there exists an ideal $B$ that $B \in G_{l . m}^{n-j}$. 
THEOREM 6.1. Let $\Lambda$ be an h-order over a discrete, rank one valuation ring $R$ in $\Sigma$. Let $\Omega_{l, m}^{n-j}, G_{l, m}^{n-j}$ be as above. Then the set of two-sided fractional ideals with respect to $\Lambda$ is a groupoid $\left({ }^{5}\right)$ with $G_{l, m}^{n-j}$ and $\Omega_{l}^{n-j}$ as unit element with respect to characteristic product. Furthermore, $G_{l, l}^{n-j}$ is a cyclic group generated by the radical $N_{l}^{(n-j)}$ of $\Omega_{l}^{n-j}$.

Proof. We have observed the first half in the above. We denote $G_{l, l}^{n-j}$, $\Omega_{l}^{n-j}$ by $G, \Omega$. $G$ is, by Lemma 1.1 , the set of two-sided ideals with respect to $\Omega$ such that $\tau_{\Omega}^{l}(A)=\tau_{\Omega}^{r}(A)=\Omega$. Hence, it consists of inversible ideals with respect to $\Omega$. Therefore, $G$ is a group. We denote $N_{l}^{(n-j)}$ by $N$. Then $N \in G$ by Theorem 5.3. Let $A \in G$ such that $A \subseteq \Lambda$ and $A \nsubseteq N$. We assume that $R$ is complete. Then there exists an idempotent element $e$ in $A+N$, and hence, $e \in \bigcap(A+N)^{n}$ $\subseteq \bigcap\left(A+N^{n}\right)=A$. Therefore, $A^{n}$ is idempotent for some $n$. If $R$ is not complete, then we can use the same argument as in the proof of Lemma 3.2. Since $A^{n} \in G$, $A=\Omega$. We have proved that $N$ is a maximal two-sided integral ideal in $G$. For any integral ideal $B$ in $G$, we can find an integer $t$ such that $N^{t} \supseteq B$ and $N^{t+1} \nsucceq B$. Then since $N^{-t} B(\subseteq \Omega)$ is not contained in $N, N^{-t} B=\Omega$. Therefore, $B=N^{t}$.

7. $H$-orders over a Dedekind ring. In the previous sections, we have studied $h$-orders over a discrete, rank one valuation ring. Now, in this section, we shall deduce properties of $h$-orders over a Dedekind domain from results in the previous sections.

Let $R$ be a Dedekind domain with field of quotients $K$ and $\Sigma$ a central simple $K$-algebra. Let $\Gamma_{1}$ and $\Gamma_{2}$ be orders containing an $h$-order $\Lambda$ over $R$, and $\Omega_{1}$ a maximal order containing $\Gamma_{1}$. Let $d=C_{\Gamma_{2}}\left(\Gamma_{1}\right) \cap R=\left\{x \mid \in R, \Gamma_{1} x \subseteq \Gamma_{2}\right\}$ and $c=C\left(\Omega_{1}\right) \cap R$; then we have $d \supseteq c$. By using prime factors $p_{1}, \cdots, p_{r}$ of $c$, we obtain a multiplicative system $S=R-\left(p_{1} \cup \cdots \cup p_{r}\right)$ in $R$. Then $d_{s}=C_{\Gamma_{2} s}\left(\Gamma_{1 s}\right)$ $\cap R_{S} \neq R_{S}$ if $d \neq R$. Hence, if $\Gamma_{1} \ddagger \Gamma_{2}$, then $\Gamma_{1 S} \ddagger \Gamma_{2 S}$. On the other hand, let $\Gamma^{\prime}$ be an order over $R_{S}$ containing $\Lambda_{S}$. Then $\Gamma^{\prime}=\operatorname{Hom}_{\Lambda_{S}}^{r}\left(E^{\prime}, E^{\prime}\right)$ for an idempotent ideal $E^{\prime}$ in $\Lambda_{S}$. Let $E=E^{\prime} \cap \Lambda$, then $\Gamma=\operatorname{Hom}_{\Lambda}^{r}(E, E)$ is an order containing $\Lambda$ such that $\Gamma_{S}=\Gamma^{\prime}$.

It is clear by Theorem 1.7 that $C(\Omega)$ is a minimal idempotent ideal in $\Lambda$ and that the set $\left\{p_{1}, \cdots, p_{r}\right\}$ does depend only on $\Lambda$, not on $\Omega$. We say that the $p_{i}$ 's belong to $\Lambda$.

Summarizing the above observation, we have

Proposition 7.1. Let $\Lambda$ be an h-order over a Dedekind domain $R$ in $\Sigma$, and let the set $\left\{p_{i}\right\}$ belong to $\Lambda$. Then there is a one-to-one correspondence between orders over $R$ containing $\Lambda$ and orders over $R_{S}$ containing $\Lambda_{S}$, which preserves the inclusion, where $S=R-\left(p_{1} \cup \cdots \cup p_{r}\right)$. 
From this proposition, we may restrict ourselves to the case where $R$ is a semi-local Dedekind domain with maximal ideals $p_{1}, \cdots, p_{r}$. Furthermore, we may assume, by the above argument, that $\Lambda_{p_{i}}$ is not maximal for each $p_{i}$. For a while we assume $R$ is semi-local. Let $n$ and $N$ be the radicals of $R$ and $\Lambda$, respectively; then $\Lambda / N=\Lambda / N \otimes_{R} R / n=\Lambda / N \otimes_{R} R / p_{1} \oplus \cdots \oplus \Lambda / N \otimes_{R} R / p_{r}$, and $\Lambda / N \otimes_{R} R / p_{i}=\Lambda_{p_{i}} / N_{p_{i}}$. On the other hand, we have $\hat{\Lambda}=\Lambda \otimes_{R} \hat{R}=\Lambda \otimes_{R} \hat{R}_{p_{1}}$ $\oplus \cdots \oplus \Lambda \otimes_{R} \hat{R}_{p_{i}}$ and $\Lambda / N=\hat{\Lambda} / \hat{N}=\Lambda / N \otimes_{R} \hat{R}_{p_{1}} \oplus \cdots \oplus \Lambda / N \otimes_{R} \hat{R}_{p_{r}}=\Lambda_{p_{i}} / N_{p_{1}}$ $\otimes_{R} \hat{R}_{p_{1}} \oplus \cdots \oplus \Lambda_{p_{r}} / N_{p_{r}} \otimes_{R} \hat{R}_{p_{r}}$, where $\hat{R}$ and the $\hat{R}_{p}$ are completions of $R$ and $R_{p}$ with respect to $n$ and $p R_{p}$, respectively. Let $A$ be a nonzero idempotent ideal in $\Lambda$; then the $((A+N) \hat{\wedge} / \hat{N}) \otimes \hat{R}_{p_{i}}$ are nonzero ideals in $\Lambda_{p_{i}} / N_{p_{i}} \otimes \hat{R}_{p_{i}}$. Conversely, if we take nonzero ideals $C_{i}^{\prime}$ in $\Lambda / N \otimes \hat{R}_{p_{i}}$ for each $i$, we can find idempotent ideals $C_{i}$ in $\hat{\Lambda}_{p_{i}}$ such that $C_{i} \equiv C_{i}^{\prime} \bmod \hat{N}_{p_{i}}$. Hence, $C=\sum_{i=1}^{r} C_{i}$ is an idempotent ideal in $\hat{\Lambda}$ such that $C+\hat{N}=\Sigma C_{i}^{\prime}\left(=C^{\prime}\right)$. However, since $\hat{\Lambda} / C$ $=\Sigma \hat{\Lambda}_{p_{i}} / C_{i}, \hat{\Lambda} / C$ satisfies the minimal condition, $C^{\prime n}$ is idempotent and $C^{\prime n}+\hat{N}=C+\hat{N}$ for some $n$. Since $C^{\prime} \not{\jmath} \hat{N}$, there exists an ideal $A$ in $\Lambda$ such that $\hat{A}=C^{\prime}$. Hence, $A^{n}$ is idempotent and $\hat{A}_{p_{i}}^{n} \equiv C_{i}^{\prime} \bmod \hat{N}_{p_{i}}$. Therefore, by Lemma 2.4, there is a one-to-one correspondence between idempotent ideals in $\Lambda$ and ideals $A$ in $\Lambda / N$ such that $A_{p_{i}} / N_{p_{i}} \neq(0)$ for all $p_{i}$. Furthermore, from the assumption that the $\Lambda_{p_{i}}$ 's are not maximal, every $\Lambda_{p_{i}} / N_{p_{i}}$ is not a simple ring.

We shall come back again to the case where $R$ is a Dedekind domain (not necessarily semi-local).

Let $\left\{p_{1}, \cdots, p_{r}\right\}$ be the set of prime ideals in $R$ which belong to an $h$-order $\Lambda$. For the set $S_{i}=R-p_{i}$, by $\rho(i)$ we denote the number of two-sided ideals $P_{i, j}$ which is a maximal ideal among the set of two-sided ideals $A$ such that $A \cap S_{i}=\phi$. Then we have a one-to-one correspondence between maximal ideals in $\Lambda_{s_{i}}$, and $P_{i, j}$. Hence, $\rho(i)$ is the number of simple components of $\Lambda_{p_{i}} / N_{p_{i}}$ which is a finite integer $\geqq 2$. Furthermore, it is clear by Theorem 1.7 that for the conductor $C$ of a maximal order containing $\Lambda$, we obtain uniquely a simple component $C_{p}+N_{p} / N_{p}$ of $\Lambda_{p} / N_{p}$ for each $p$ and conversely. Similarly, for the conductor $C^{\prime}$ of a minimal order containing $\Lambda$, we can find uniquely a prime ideal $p$ in $R$ and a maximal ideal $\bar{A}_{p}$ in $\Lambda_{p} / N_{p}$ such that $C_{p}^{\prime}+N_{p} / N_{p}=\bar{A}_{p}$ and $C_{q}^{\prime}+N_{q} / N_{q}=\Lambda_{q} / N_{q}$ if $p \neq q$.

Using the above observations and Proposition 3.1 we have a generalization of Theorem 3.3.

THEOREM 7.2. Let $\Lambda$ be an h-order over a Dedekind domain $R$ in $\Sigma$. Let $\rho(i)(i=1, \cdots, r)$ be as above. Then there are precisely $\Pi_{i} \rho(i)$ maximal order $\Omega_{i}$ containing $\Lambda$ and $\Sigma_{i} \rho(i)$ minimal orders $\Gamma_{i, j}(i=1, \cdots, r ; j=1, \cdots, \rho(i))$, containing $\Lambda$. For an order $\Gamma(\underset{\neq}{\supset} \Lambda)$, we have a unique expression of $\Gamma: \Gamma=$ $\bigcup_{i=1}^{\rho \leqq r} \bigcup_{k=1}^{t i<p(l)} \Gamma_{l, j k_{l}}$ and for order $\Gamma^{\prime}(\supseteq \Lambda), \Gamma^{\prime}=\bigcap_{i} \Omega_{i}$ (not necessarily unique) where $\Omega_{i}$ runs through all maximal orders containing $\Lambda$. Consequently, the number of orders containing $\Lambda$ is equal to $\prod_{i=1}^{r}\left(2^{\rho(i)}-1\right)$. 
Let $C_{1}$ and $C_{2}$ be idempotent ideals in $\Lambda$, then we shall say that $C_{1}$ and $C_{2}$ belong to the same type if $C_{1 p}+N_{p} / N_{p}$ and $C_{2 p}+N_{p} / N_{p}$ have the same number of simple components for each $p$.

If $\Gamma=\bigcup \bigcup \Gamma_{l, j k_{l}}$ and $C=C(\Gamma)$, then $C+N / N$ is isomorphic to a unique decomposition: $\sum_{l=1}^{s} \sum_{m=1}^{\rho_{l}-t_{l}} \hat{A}_{l, j m}+\sum_{l=s+1}^{r} \Lambda_{p l} / N_{p l}$, where $\hat{A}_{l, j}$ is a simple component of $\Lambda_{p l} / N_{p l}$. We call $\Gamma$ an sth order.

If $\Gamma$ is a 1 st order, then we have the same situation as in the previous section. Therefore, we can prove the following theorem by induction on $s$ as in the proof of Theorem 4.3 .

THEOREM 7.3. Let $\Lambda$ be as above, and $\Gamma_{1}, \Gamma_{2}$ orders containing $\Lambda$. Then we have the following equivalent conditions:

(1) $\Gamma_{1}$ and $\Gamma_{2}$ belong to the same type.

(2) $\Gamma_{1 p}$ and $\Gamma_{2 p}$ belong to the same type for each prime ideal $p$ in $R$.

(3) $C\left(\Gamma_{1}\right)$ and $C\left(\Gamma_{2}\right)$ belong to the same type.

Finally, we consider a group structure of two-sided ideals with respect to $\Lambda$. We use the following well-known lemma:

Lemma 7.4. Let $E$ be a finitely generated $R$-module and $A, B$ submodule in $E$. Then we have $(A: B)_{p}=\left(A_{p}: B_{p}\right)$ for every prime ideal $p$ in $R$.

LEMMA 7.5. For each prime ideal $p$ in $R$, there exists a two-sided ideal $N(p)$ such that $N(p)_{p}$ is the radical of $\Lambda_{p}$ and $N(p)_{q}=\Lambda_{q}$ if $p \neq q$.

Proof. Let $R\left(\Lambda_{q}\right)$ be the radical of $\Lambda_{q}$ and $N(p)=R\left(\Lambda_{p}\right) \cap \Lambda$. Then $N(p)_{p}=R\left(\Lambda_{p}\right)$. Furthermore, since $N(p) \supseteq R\left(\Lambda_{p}\right) \cap \Lambda \cap R \supseteq p \Lambda \cap R=p, N(p)_{q} \supseteq p_{q}=R_{q}$ if $p \neq q$.

Thus, we have the following generalization of $[7$, p. 74, Satz 9].

THEOREM 7.6. Let $\Lambda$ be an h-order over a Dedekind domain $R$ in $\Sigma$. Then the set of inversible two-sided ideals $A$ with respect to $\Lambda$ is an abelian group which is a direct product of cyclic groups generated by $N(p)$.

Proof. It is clear that $A$ is an inversible ideal if and only if $\tau_{\Lambda}^{l}(A)=\tau_{\Lambda}^{r}(A)=\Lambda$. By Lemmas 7.4 and 7.5, and Theorem 6.1, we have $\tau_{\Lambda}^{l}(N(p))=\tau_{\Lambda}^{r}(N(p))=\Lambda$ and hence, $N(p)$ is inversible. Let $A$ be an inversible ideal with respect to $\Lambda$. Then $A_{p}=R\left(\Lambda_{p}\right)^{\rho(p)}$ by Theorem 6.1, and the $\rho(p)$ are equal to zero except a finite number of $p$. Hence, by Lemmas 7.4 and 7.5, we have $A=\prod N(p)^{\rho(p)}$. Furthermore, $(N(p) N(q))_{r}=(N(q) N(p))_{r}$ for every prime ideal $r$ in $R$. Thus, we prove the theorem.

REMARK 3. We can construct a groupoid structure of two-sided ideals with respect to $\Lambda$ as in Theorem 6.1. 


\section{BIBLIOGRAPHY}

1. E. Artin, Zur Arithmetik hyperkomplexer Zahlen, Abh. Math. Sem. Univ. Hamburg 5 (1928), 261-289.

2. K. Asano, Theory of rings and ideals, Tokyo, 1949.

2'. — Arithmetische Idealtheorie in nicht kommutative Ringen, Japan. J. Math. 16 (1939), 1-36.

3. M. Auslander and O. Goldman, Maximal orders, Trans. Amer. Math. Soc.97(1960),1-24.

4. - The Brauer group of a commutative ring, Trans. Amer. Math. Soc. 97 (1960), 367-409.

5. H. Cartan and S. Eilenberg, Homological algebra, Princeton Univ. Press, Princeton, N. J., 1957.

6. M. Deuring, Algebren, Springer, Berlin, 1935.

7. S. Eilenberg, Homological dimension and syzygies, Ann. of Math. (2) 64 (1956), 328-336.

8. M. Harada, Note on raising idempotents, J. Inst. Polytech. Osaka City Univ. Ser. A 10 (1959), 63-65.

9. - Hereditary orders in quaternions, J. Inst. Polytech. Osaka City Univ., (to appear).

10, K. Henke, Zur arithmetischen Idealtheorie hyperkomplexer Zahlen, Abh. Math. Sem. Univ. Hamburg 11 (1935), 311-332.

11. P. Samuel and O. Zarisky, Commutative algebra. II, Princeton, N. J., 1960.

NORTHWESTERN UNIVERSITY,

EVANSTON, ILLINOIS

OSAKa CITY University,

OSAKA, JAPAN. 